\title{
Editorial: Antibiotic Alternatives and Combinational Therapies for Bacterial Infections
}

\author{
Sanna Sillankorva*, Maria Olívia Pereira and Mariana Henriques \\ Laboratório de Investigação em Biofilmes Rosário Oliveira, Centre of Biological Engineering, University of Minho, \\ Braga, Portugal
}

Keywords: antibiotic alternatives, bacterial infection, probiotic, bacteriophage, anti-persister molecule, biofilm

\section{Editorial on the Research Topic}

\section{Antibiotic Alternatives and Combinational Therapies for Bacterial Infections}

\section{OPEN ACCESS}

Edited by:

Stephen Tobias Abedon The Ohio State University,

United States

Reviewed by:

Sarah J. Kuhl,

VA Northern California Health Care

System, United States

Beata Weber-Dąbrowska,

Institute of Immunology and

Experimental Therapy (PAN), Poland

*Correspondence:

Sanna Sillankorva s.sillankorva@deb.uminho.pt

Specialty section

This article was submitted to Antimicrobials, Resistance and Chemotherapy,

a section of the journal

Frontiers in Microbiology

Received: 10 October 2018 Accepted: 31 December 2018 Published: 18 January 2019

Citation:

Sillankorva S, Pereira MO and Henriques M (2019) Editorial: Antibiotic Alternatives and Combinational Therapies for Bacterial Infections. Front. Microbiol. 9:3359. doi: 10.3389/fmicb.2018.03359
"The thoughtless person playing with penicillin treatment is morally responsible for the death of the man who succumbs to infection with the penicillin-resistant organism." As Alexander Fleming predicted in 1945, bacteria have become increasingly resistant to antibiotics. Penicillin resistance was presumably first reported already in 1940 when Abraham and Chain reported that an enzyme from bacteria was able to destroy penicillin (Abraham and Chain, 1940). Every now and then mankind is shelled with news of infections and deaths caused by antibiotic and multiple drug resistant superbugs. This increase of resistance toward commonly in-use antibiotics, due to decades of their use, misuse and abuse, is today a global health concern. Research investments on development of new agents that can fight antimicrobial resistant microorganisms and the advent of antibiotic failure due to bacterial resistance has raised interest in other non-conventional alternative therapies.

This Research Topic gathers some of the latest science around antibiotic alternatives and the effect of combined therapies. The call was launched in July 2017, and open-call papers were submitted until May 2018. This is the editorial article introducing the 20 accepted publications addressing the antimicrobial action of varied agents representing the breadth and scope of research in this topic.

A high number of publications address the antibacterial use of bacteriophages. A mini review by Morozova et al. describes the main outcomes of English and Russian case reports regarding bacteriophage use in infected wounds, burns and trophic ulcers. The antimicrobial assessment of bacteriophage therapy include in vitro testing toward biofilms of Klebsiella pneumoniae isolated from diabetic foot patients (Taha et al.), Staphylococcus aureus biofilms (Kumaran et al.), and their combined use with honey to control dual species biofilms of Pseudomonas aeruginosa and Escherichia coli in an ex vivo wound model (Oliveira et al.). Overall, bacteriophages were able to decrease bacterial loads and destroy biofilm structures. Bacteriophage-antibiotic treatment order was investigated by Kumaran et al. and this greatly influenced the treatment outcome, and bacteriophages always augmented the activity of antibiotics. Ujmajuridze et al. screened cultures of patients planned for transurethral resection of prostate, adapted the commercial Pyo bacteriophage preparation to target the main species identified (S. aureus, E. coli, Streptococcus spp., P. aeruginosa, and Proteus mirabilis), administered the preparation via intravesical delivery in nine patients, and observed bacterial decrease in six of the nine patients treated. In vivo use of a purified bacteriophage capsule depolymerase to treat E. coli infections in a mouse thigh model was also studied (Lin et al.). In this work, the authors show that E. coli infections, usually lethal to mice, were effectively treated with an 
enzyme dose of $20 \mu \mathrm{g}$ per mouse; however this effect was enzyme and capsule type dependent.

Three original research articles assessed the use of probiotics such as Lactobacillus plantarum or L. rhamnosus. Wang et al. describe the diverse roles of $L$. plantarum from enhancing the intestinal barrier function, inducing the secretion of antimicrobial peptides that protect against pathogens, improving the gut bacterial ecology and barrier function in weaned piglets. The efficacy of $L$. plantarum in preventing enterotoxigenic $E$. coli growth and inhibiting its adhesion to a porcine intestinal epithelial cell line was also assessed (Wang et al.). L. rhamnosus was reported to reduce the adhesion of $E$. coli to bovine mammary epithelial cells devoid of the caspase recruitment domain by supressing the NLRP3 and NLRP4 inflammasomes and inhibiting E. coli-induced cell pyroptosis (Wu et al.).

Defraine and colleagues reported extensive $P$. aeruginosa membrane damage caused by a novel anti-persister molecule (Defraine et al.) and its antibacterial effect together with different classes of antibiotics toward clinically relevant ESKAPE pathogens (Defraine et al.). The molecule used (SPI009) has great potential to inhibit biofilm growth and eradicate both $P$. aeruginosa and $S$. aureus biofilms, and improved nematode survival when tested in Caenorhabditis elegans infected with $P$. aeruginosa. Vitamin $\mathrm{C}$ was shown to have antibiofilm activity against Bacillus subtilis by reducing the extracellular polymeric substance biosynthesis, with cells becoming more susceptible for killing (Pandit et al.).

An original article by Klitgaard et al. identified potential genes that could be suitable as targets for ciprofloxacin potentiating compounds, and found that in targeting the AcrAB-TolC efflux pump and the SOS response proteins RecA and RecC, $E$. coli resistance to ciprofloxacin was reverted in intermediate susceptible strains.

Antibiotic derivatives were reported by Ramchuran et al., who used three teixobactin derivatives to inhibit methicillinresistant $S$. aureus (MRSA) growth, giving evidence of its dominant binding mode to lipid II. Antibiotic combinations against established $S$. aureus biofilms were also studied in a hollow fiber infection model. However, no beneficial effect of combination therapy compared to the most effective antibiotic was observed, though the addition of the second antibiotic reduced the rise of bacterial resistant to the first drug (Broussou

\section{REFERENCES}

Abraham, E. P., and Chain, E. (1940). An Enzyme from bacteria able to destroy penicillin. Nature 146:837. doi: 10.1038/146837a0

Conflict of Interest Statement: The authors declare that the research was conducted in the absence of any commercial or financial relationships that could be construed as a potential conflict of interest. et al.). Anti-MRSA activity using cationic nanostructured lipid carriers combined with antibiotic was evaluated in mice models of cutaneous infection resulting in infection reduction and improvement of skin barrier function and architecture (Alalaiwe et al.). The topical efficacy and safety of chitogel assembled together with an iron chelator and with a novel broad spectrum antimicrobial effectively reduced $S$. aureus biofilms in an in vivo sheep model without causing any topical or systemic adverse effects (Ooi et al.). The topical treatment of recalcitrant chronic rhinosinusitis using colloidal silver was assessed through a 10day program where patients performed rinsing twice daily (Ooi et al.). Despite being safe, the group of treated patients had similar improvement in symptoms and endoscopic scores as those in the control groups and were inferior to culture-directed oral antibiotics. Tran et al. used an antineoplastic mitotane, that permeabilize the outer membrane of $P$. aeruginosa, Acinetobacter baumannii and K. pneumoniae, to exert greater effect to a novel polymyxin, and reduce the emergence of antibiotic-resistant phenotypes.

Three branched RRWQWR-based cationic peptides were designed, synthesized and evaluated revealing higher antibacterial activity against clinically relevant pathogens than the reference peptide (Vega et al.).

We hope that you enjoy reading this Research Topic and find it a useful reference for the state of the art in the emerging field of antibiotic alternatives.

\section{AUTHOR CONTRIBUTIONS}

All authors listed have made a substantial, direct and intellectual contribution to the work, and approved it for publication.

\section{FUNDING}

This study was supported by the Portuguese Foundation for Science and Technology (FCT) under the scope of the strategic funding of UID/BIO/04469 unit and COMPETE 2020 (POCI01-0145-FEDER-006684) and BioTecNorte operation (NORTE01-0145-FEDER-000004) funded by the European Regional Development Fund under the scope of Norte2020-Programa Operacional Regional do Norte. SS is Investigador FCT (IF/01413/2013).
Copyright (C) 2019 Sillankorva, Pereira and Henriques. This is an open-access article distributed under the terms of the Creative Commons Attribution License (CC BY). The use, distribution or reproduction in other forums is permitted, provided the original author(s) and the copyright owner(s) are credited and that the original publication in this journal is cited, in accordance with accepted academic practice. No use, distribution or reproduction is permitted which does not comply with these terms. 